PALEO

Revue d'archéologie préhistorique

19 | 2007

Spécial table ronde ( 1 ère partie) : Le Gravettien : entités régionales d'une paléoculture européenne, Les Eyzies, juillet 2004

\title{
Approche stratigraphique des temps Gravettiens : l'éclairage aquitain
}

A Stratigraphic approach to the Gravettian: a view from the Aquitaine.

Françoise Delpech et Jean-Pierre Texier

CpenEdition

Journals

Édition électronique

URL : https://journals.openedition.org/paleo/487

DOI : $10.4000 /$ paleo.487

ISSN : $2101-0420$

Éditeur

SAMRA

Édition imprimée

Date de publication : 30 décembre 2007

Pagination : 15-29

ISSN : $1145-3370$

Référence électronique

Françoise Delpech et Jean-Pierre Texier, «Approche stratigraphique des temps Gravettiens

l'éclairage aquitain », PALEO [En ligne], 19 | 2007, mis en ligne le 23 avril 2009, consulté le 25 juin 2021. URL : http://journals.openedition.org/paleo/487 ; DOI : https://doi.org/10.4000/paleo.487

\section{(c) (i) $\odot$}

PALEO est mis à disposition selon les termes de la licence Creative Commons Attribution - Pas d'Utilisation Commerciale - Pas de Modification 4.0 International. 


\title{
APPROCHE STRATIGRAPHIQUE DES TEMPS GRAVETTIENS : L'ÉCLAIRAGE AQUITAIN
}

\author{
Françoise DELPECH ${ }^{(1)}$ et Jean-Pierre TEXIER ${ }^{(1)}$
}

\begin{abstract}
Résumé : Le cadre spatio-temporel du gravettien est relativement large. En effet, la période gravettienne a sans doute duré plusieurs milliers d'années, au moins 6000 " années ${ }^{14} \mathrm{C}$ " réparties de part et d'autre de 25000 BP. Quant au territoire, il s'étale d'ouest en est sur toute l'Europe, ce qui pose la question des corrélations à grande distance. Dans ce travail, nous avons utilisé les moyens et méthodes de la stratigraphie pour tenter d'établir des liens chronologiques entre entités gravettiennes de l'ouest et du centre de l'Europe. Pour cela, nous nous sommes intéressés tout d'abord à la région Aquitaine et avons recherché des phénomènes environnementaux marqueurs. Ces derniers ont servi de points d'ancrage entre entités stratigraphiques ouest et centre européennes. Les datations physiques, prises en compte dans un deuxième temps, ne contredisent pas l'hypothèse de corrélation proposée. Elles conduisent néanmoins à mettre en garde contre l'utilisation d'une maille chronologique trop fine.
\end{abstract}

Mots-clés : Gravettien, environnements, stratigraphie, liaisons chronologiques sur de grandes distances.

Abstract : A Stratigraphic Approach to the Gravettian: a View from the Aquitaine. The Gravettian was widely distributed in time and space. Temporally, it lasted at least 6000 radiocarbon years, centering on 25,000 BP. Spatially, it extended across all of Europe, posing the challenge of correlating assemblages across vast distances. We have used stratigraphic methods in an attempt to establish chronological ties between Gravettian assemblages in western and central Europe. Using the record from the Aquitaine, we have constructed a series of environmental horizon markers which serve as anchor points among stratigraphic entities in western and central Europe. Our hypothesized correlations are not contradicted by the radiocarbon dates available for the Gravettian. Those dates do, however, suggest that we should be wary of building highly precise links among far-flung Gravettian assemblages.

Key-Words : Gravettian, environments, stratigraphy, chronological ties across large distances.

\section{INTRODUCTION}

Le titre même du colloque organisé par J.-Ph. Rigaud du 7 au 9 juillet 2004 aux Eyzies-de-Tayac en Dordogne : "Entités régionales d'une paléoculture européenne, le Gravettien " invite à réfléchir sur un Gravettien non monolithique dont les expressions (notamment techno-typologiques) peuvent varier selon les régions. Or, le Gravettien s'est sans doute développé durant plusieurs millénaires et son extension spatiale concerne un territoire aussi vaste que celui de la péninsule européenne. Si la répartition géographique des données relatives à cette culture est bien connue (puisqu'elle coïncide avec celle des sites), il n'en est pas de même de leur situation chronologique et, surtout, de leur âge relatif. Or, pour interpréter les différences mises en évidence, pour comprendre le sens des variations rencontrées (régionales donc géographiques ou (et) temporelles), pour élaborer des hypothèses sur l'écologie humaine, sur l'évolution des comportements ou du degré de cognition des hommes du passé, les données étudiées doivent avoir été classées dans un ordre chronologique. C'est ce que nous nous sommes attachés à faire ici en respectant scrupuleusement les règles et les principes de la stratigraphie (Hedberg 1979). Ce classement a ensuite servi de base à l'établissement de la succession des paléoenvironnements et à la caractérisation d'événements marqueurs à grande portée géographique dont la définition est donnée plus loin.

Dans le développement qui suit, on replacera d'abord les différentes phases du Gravettien d'Europe occidentale dans leur contexte environnemental puis, en s'appuyant sur des événements marqueurs, on essaiera de les situer par rapport à celles identifiées en Europe centrale. Dans cette zone géographique, les sites gravettiens, associés la plupart du temps à des dépôts lœssiques, ont fait récemment l'objet de travaux de synthèse (Haesaerts et al. 2003, 2004). 
Notre réflexion ne se limitera donc pas à l'Aquitaine comme le titre de l'article semble l'indiquer. En choisissant une annonce apparemment réductrice, nous avons voulu insister sur l'importance de considérer en priorité des entités régionales cohérentes à partir desquelles des successions environnementales fiables peuvent être établies.

\section{ENVIRONNEMENTS BIOLOGIQUES ET CONDITIONS CLIMATIQUES}

On sait que les environnements biologiques varient avec le climat. Le seul fait qu'il existe actuellement une zonation des biocœnoses qui coïncide assez précisément avec celle des aires climatiques, permet de justifier cette affirmation. Ce phénomène, que l'on peut facilement percevoir aujourd'hui en se déplaçant du sud au nord ou en s'élevant en altitude, s'inscrit également dans le temps : " II existe une remarquable analogie entre la succession des biocœnoses dans le temps... et celle observée ...dans un écocline " écrit François Ramade (1984 p. 295). En conséquence, quand on se place dans une aire géographique donnée, il est possible non seulement de percevoir les changements de biocœnoses, donc les variations climatiques qui se sont succédé au cours du temps, mais aussi d'apporter des arguments sur le sens des flux migratoires qui ont présidé à ces changements (Delpech 1999 et 2008).
Pour lire les variations climatiques, du moins pour avoir une idée du nombre et de l'importance des changements, il est nécessaire de s'appuyer sur une séquence stratigraphique de référence qui englobe l'ensemble de la période concernée. S'il n'y a pratiquement aucune chance pour qu'un seul site livre une séquence reflétant la totalité des évènements, il est possible, en revanche, d'établir une biostratigraphie de référence à partir des données biologiques issues de plusieurs gisements. Cette séquence biostratigraphique, qui pourra être complétée au fil des découvertes, sera propre à la région étudiée. Néanmoins, comme rappelé plus haut, elle sera également susceptible de nous renseigner sur des phénomènes climatiques et environnementaux touchant des régions avoisinantes voire plus lointaines.

\section{LE CAS DE L'AQUITAINE SEPTENTRIONALE}

La zone géographique choisie est la région Aquitaine. Située au sud ouest de la plaine nord-européenne, l'Aquitaine a pu (dû) enregistrer assez fidèlement des phénomènes propres à l'ensemble de l'Europe du nord et de l'est, voire à l'ensemble de l'Europe non méditerranéenne, l'Europe méditerranéenne constituant, aujourd'hui comme par le passé, un autre monde (Delpech 1999, 2003).

En Aquitaine, la biostratigraphie relative à la tranche de temps contemporaine des techno-complexes du

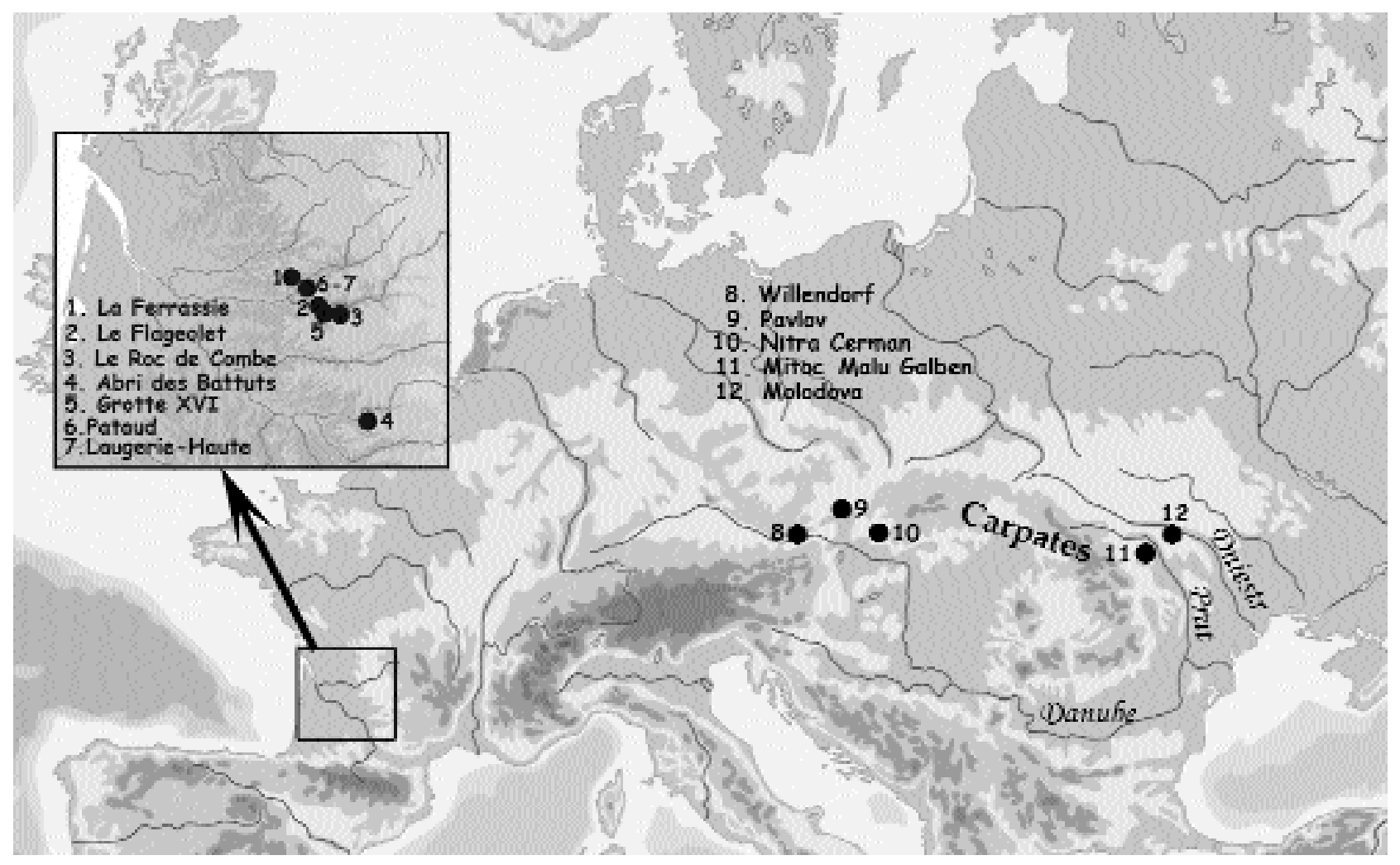

Figure 1 - Localisation géographique des gisements gravettiens cités dans le texte.

Figure 1 - Locations of Gravettian sites discussed in the text. 


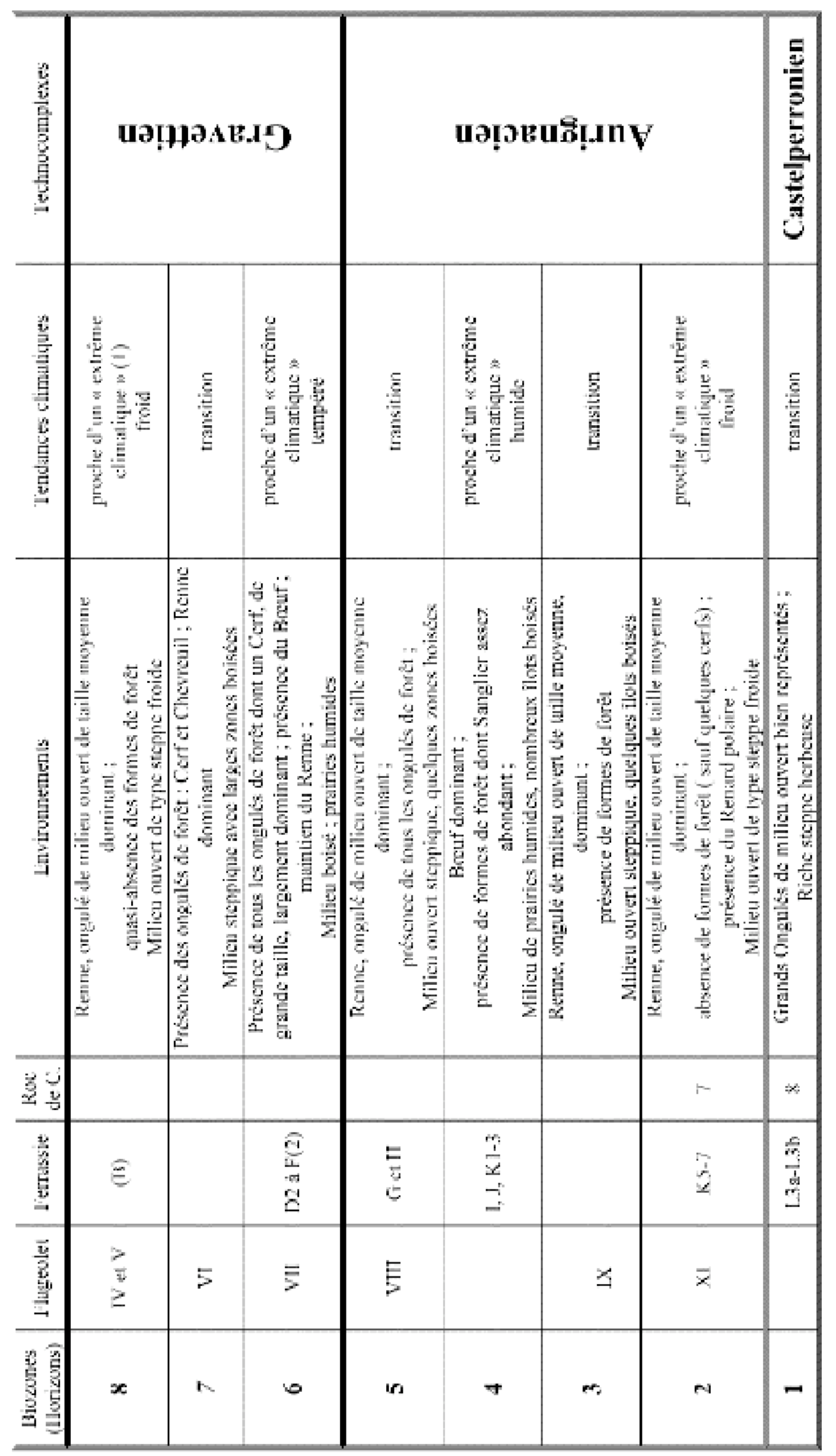

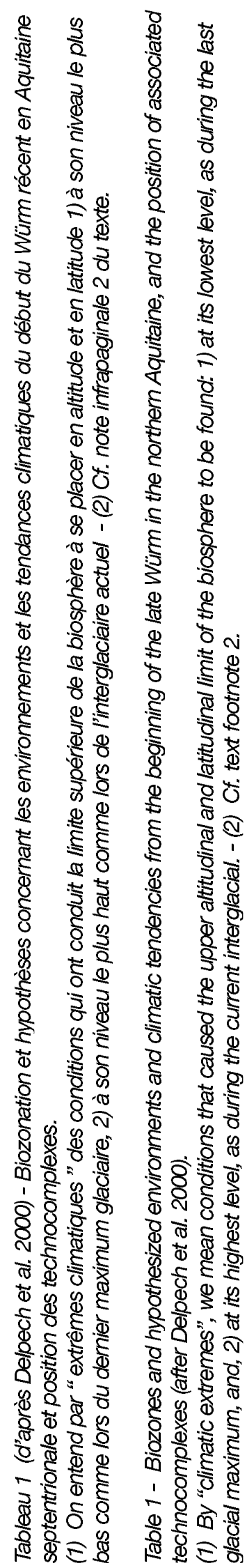


Paléolithique supérieur a été établie à partir des données foumies par les grands mammifères de trois gisements: le Roc de Combe (à Payrignac dans le Lot), la Ferrassie (à Savignac de Miremont en Dordogne) et Le Flageolet I (à Bézenac en Dordogne) (fig. 1). Les ensembles stratigraphiques concernés se sont formés au cours d'une période qui a vu l'apparition puis le développement des technocomplexes castelperroniens, aurignaciens et gravettiens. Toute une succession d'événements climatiques a été mise en évidence (Delpech et al. 2000) au sein de laquelle il est possible de situer les diverses manifestations du Gravettien. A partir de ces trois gisements, huit ensembles stratigraphiques ont été définis d'un point de vue biologique. Ils correspondent à autant de zones climatiques et de zones chronologiques dont les caractéristiques sont rappelées dans le tableau 1. Dans ce demier, on constate que les ensembles gravettiens font suite aux ensembles aurignaciens $^{2}$ et qu'ils se sont formés lors de trois périodes, la première et la troisième affichant des conditions très différentes l'une de l'autre : la première est proche d'un optimum tempéré tandis que la troisième est caractérisée par un climat très froid, proche de celui des maximums glaciaires. La deuxième période, quant à elle, correspond à une phase climatique de transition.

La période la plus ancienne a été définie à partir des couches VII du Flageolet et des couches D2 à $\mathrm{F}^{2}$ de la Ferrassie. Presque tous les ongulés y sont représentés. C'est cependant aux formes de forêt qu'une large majorité des restes se rapportent, plus particulièrement au Cerf qui est de grande taille. Le Renne est rare et, parmi les Bovinés, Bos primigenius a pu être identifié. Lynx spelaea est présent parmi les camivores. Relativement à la période précédente, les zones boisées sont sans doute plus largement étendues ce qui ne semble pas nuire au Renne dont quelques sujets, au moins, atteignent une grande taille. (Delpech et al. 2000, p. 123).

La période suivante concerne la couche VI du Flageolet. L'association faunique "indique... une réduction des zones boisées (disparition du Sanglier mais aussi du Mégacéros et d'Equus hydruntinus) qui va sans doute de pair avec un élargissement de zones steppiques (plus de Renne, présence d'Alopex lagopus, présence également de Coelodonta antiquitatis). Pour le Renne, l'environnement semble toujours favorable : certains sujets sont encore de grande taille..." (Delpech et al. op. cit., p. 123).

Dans les couches IV et V du Flageolet (et l'ensemble B de La Ferrassie) qui se sont formées postérieurement " l'association des ongulés se compose presque exclusivement des éléments de base : Renne, Bovinés (sans doute Bison), Cheval, Bouquetin et Chamois. ... Un milieu de steppe froide, déboisé, se met en place qui semble plus contraignant au Renne que précédemment. Certains sujets paraissent assez petits ce qui marque... le début de la période de contrainte (centrée sur le maximum glaciaire) qui a conduit à une diminution notoire de la taille des individus ".. (Delpech et al. op. cit., p. 123).

Ainsi, dans les deux gisements du Flageolet I et de La Ferrassie, les ensembles gravettiens font suite à des ensembles aurignaciens ; dans ces deux sites aussi, le gravettien apparaît lors d'une période relativement douce puis se développe alors que se met en place la dégradation climatique qui conduit au dernier maximum glaciaire. On retrouve ce même schéma dans un autre gisement aquitain, l'abri des Battus à Penne dans le Tarn (Alaux 1969) (fig. 1). Ce gisement n'a pas été considéré lors de l'établissement de la biozonation régionale en raison du nombre trop peu important de strates bien documentées biologiquement. Dans ce site, le gravettien apparaît lors de la formation de la couche 5 et perdure lors de la formation des couches $6,7,9$ et 12. La couche 5 surmonte les couches 3 et 2 , aurignaciennes, et s'individualise du point de vue de l'association des ongulés par le fort développement du Cerf et la quasi-disparition du Renne. Dans les couches sus-jacentes 7 et 9 qui ont livré quelques vestiges osseux, le Cerf est de nouveau rare et c'est le Renne qui domine indiquant le même renversement de tendance que celui constaté à La Ferrassie et au Flageolet I (Delpech 1983). La couche 5 se place donc au sein de la biozone 6 , les couches 7 et 9 dans l'une des (ou dans les) biozones sus-jacentes 7 et 8 (tabl. 2).

A la Grotte XVI (Cénac-et-Saint-Julien, Dordogne), seule la couche Abc livre du gravettien (Rigaud et al. 2002). Comparé à ceux plus récents qui dénotent un climat plus froid (ceux de la biozone 8) et à ceux plus anciens qui correspondent à un climat doux et humide (ceux de la biozone 6), l'ensemble faunique de la grotte XVI (Grayson et Delpech 2003) se place en intermédiaire (fig. 2) ; aussi nous avons situé la strate Abc gravettienne au niveau de la biozone 7 (représentée, jusque là par la couche $\mathrm{VI}$ du Flageolet) qui correspond à la période climatique de transition (tabl. 2).

Dans le gisement de Laugerie-Haute (Les Eyzies, Dordogne) (fig. 1), les technocomplexes qualifiés de " périgordien III " (Peyrony et Peyrony 1938, p. 12 et 20 ; Bordes, 1958 p. 212) puis de "périgordien VI" (cf. Bordes 1968 p. 60) seraient maintenant dits " gravettiens" ; de même, aussi, ceux notés " protomagdalénien " ou " périgordien VII " (Bordes et Sonneville-Bordes 1966) ${ }^{3}$. Dans les couches 38 et 40 (des fouilles F. Bordes) avec périgordien $\mathrm{VI}$ et dans la couche 36 avec protomagdalénien, les associations fauniques sont presque entièrement composées d'ongulés indiquant un milieu froid comme, d'ailleurs, dans les niveaux sus-jacents (non gravettiens) où la faune indique toujours un climat froid ; les rennes y sont de petite taille (Delpech 1983, 1986 ; Delpech 2003) et aucun élément n'indique de mouvements de faune marqueurs de

(2) La coupure entre Aurignacien et Gravettien se situe, peut-être, à La Ferrassie au-dessus de F mais nous l'avons maintenue au-dessous en raison des questions en suspens relatives à l'homogénéité des échantillons (Delpech 2007). Selon H. Delporte, la couche F livrerait une industrie aurignacienne " aurignacien IV ?". Cependant, l'auteur doute de l'homogénéité de la série qui est, en outre, assez pauvre et dont une lamelle "pou rait être considérée comme une microgravette " (Delporte 1984 p. 184).

(3) Mais ce point de vue ne fait pas encore aujourd'hui l'unanimité (cf. Rigaud 2008 et bibliographie citée). 


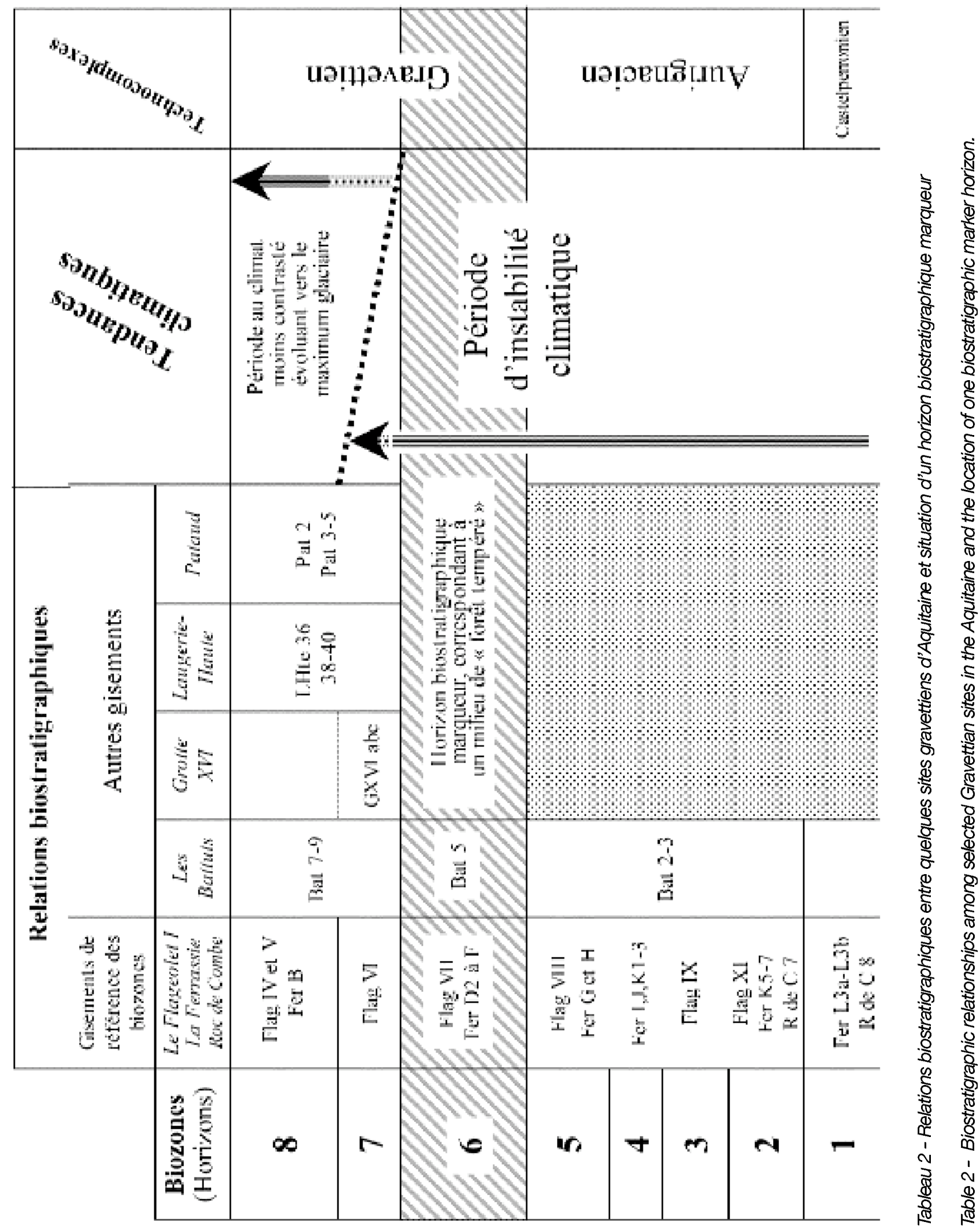




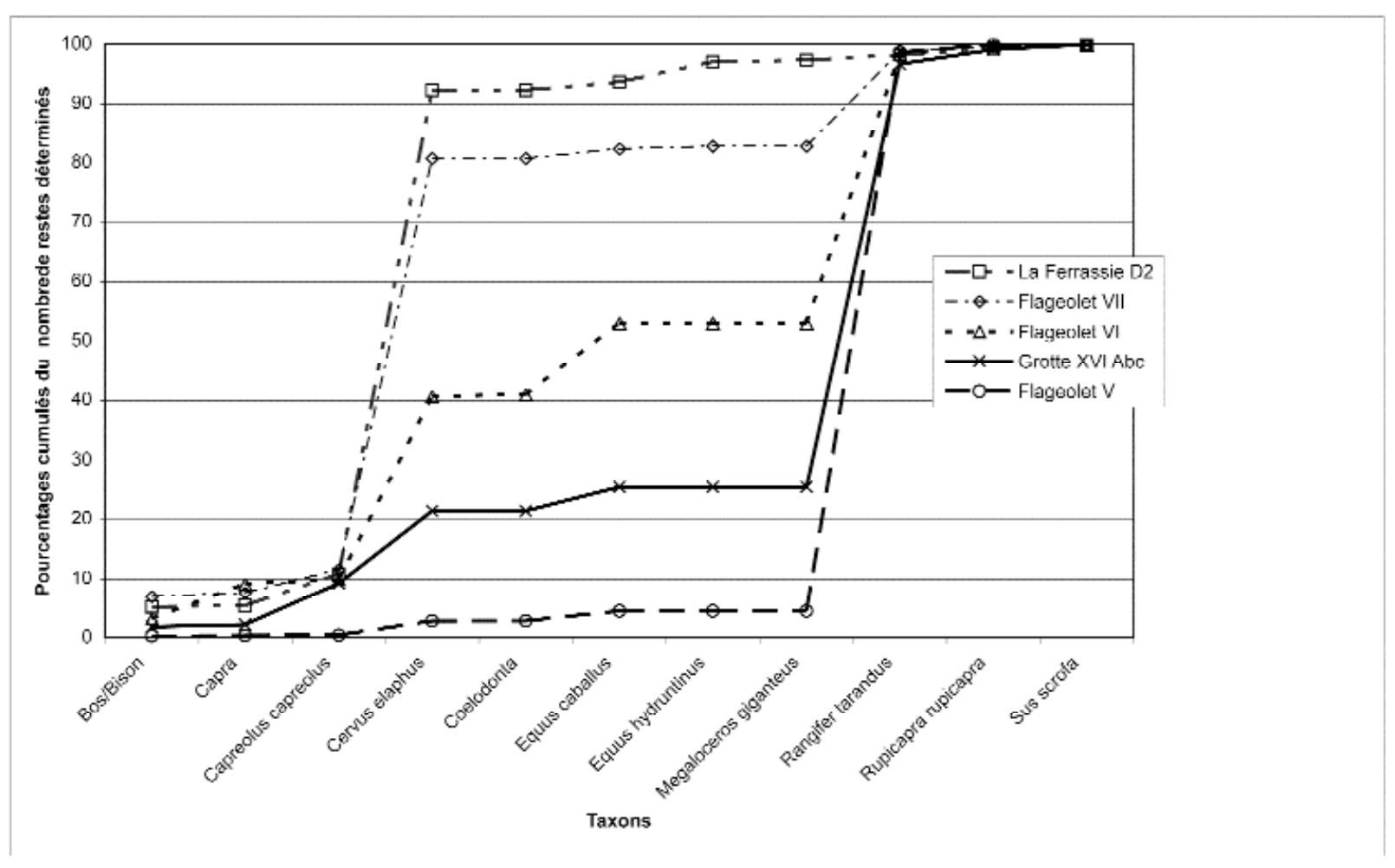

Figure 2 - Représentation graphique de quelques associations d'ongulés caractérisant les biozone 6 (ensemble VII du Flageolet I, ensemble D2 de La Ferrassie), 7 (ensemble VI du Flageolet I) et 8 (ensemble V du Flageolet I). Remarquer la position de l'ensemble Abc de la Grotte XVI entre "Flageolet $V$ " et "Flageolet VI " et la forme de la courbe le représentant, plus proche de "Flageolet VI" que de "Flageolet V"..

Figure 2 - Graphical representation of ungulate associations characteristic of biozone 6 (assemblage VII from Le Flageolet I and D2 from La Ferrassie), 7 (assemblage VI from Flageolet I), and 8 (assemblage $V$ from Flageolet I). Note the position of Grotte XVI assemblage Abc between "Flageolet V" and "Flageolet VI", and the form of the curve representing it, placing it closer to "Flageolet VI" than to "Flageolet V".

grands changements climatiques. On évolue, semble-t-il régulièrement, vers les conditions sévères du dernier maximum glaciaire.

Ainsi, les niveaux avec " Périgordien VI " et " Protomagdalénien " de Laugerie-Haute se seraient peut-être formés pro parte lors de la deuxième période, mais sûrement lors de la troisième période gravettienne définie plus haut (tabl. 2).

D'autres sites aquitains ont livré du Gravettien et l'on ne peut ignorer l'Abri Pataud (Les Eyzies, Dordogne) (fig. 1). Dans ce gisement, le Gravettien concerne les ensembles 5 à 2 (les ensembles 5 à 3 livrent du Gravettien, l'ensemble 2 du "Protomagdalénien ") et ce sont les ongulés de climat froid qui dominent largement (Bouchud 1975 ; Spiess 1979). II n'y a pas de niveau livrant une faune aussi tempérée que celle de la couche VII du Flageolet I ou des couches D2 à F de La Ferrassie. Aussi, n'ayant pas d'arguments pour scinder la séquence en deux ensembles biostratigraphiques ${ }^{4}$, nous proposons de placer les couches 5 à 2 au niveau des zones 7 et 8 , comme les couches 7 et 8 des Battuts et les couches 40 à 36 de Laugerie-Haute (tabl. 2).

\section{“ PHENOMENES MARQUEURS "}

D'après les quelques éléments précédemment exposés, le Gravettien aquitain se développe au cours de trois périodes successives et climatiquement distinctes. La première période, au climat relativement doux et humide, a sans doute favorisé la mise en place d'une forêt aux essences à feuilles caduques. L'association des ongulés est en effet suffisamment caractéristique pour justifier une telle hypothèse. La deuxième période s'écoule sous un climat que l'on peut qualifier " de transition" tandis que la troisième correspond à un environnement steppique froid (tabl. 1).

Les périodes au cours desquelles sont enregistrés les phénomènes gravettiens font suite à celles contemporaines des technocomplexes aurignaciens. Jusqu'à la fin de la première période gravettienne, les données continentales locales (Delpech et al. 2000 ; Delpech 2003 ; Laville et al. 1983), comme celles d'Europe centrale et orientale (Haesaerts et al. 2003, 2004), révèlent une forte instabilité climatique. En Aquitaine, ces conditions spécifiques qui

(4) qui auraient pu se placer respectivement sous et sur la zone 6. 


\begin{tabular}{|c|c|c|c|c|}
\hline & & \multicolumn{2}{|c|}{ Europe Centrale } & \\
\hline $\begin{array}{l}\text { "tendances } \\
\text { climatiques " }\end{array}$ & «Phénomènes marqueurs» & & $\begin{array}{l}\text { Ouest des } \\
\text { Carpates }\end{array}$ & \\
\hline $\begin{array}{l}\text { Refroidissement } \\
\text { drastique }\end{array}$ & " épais gley de toundra " & $\begin{array}{l}\text { affecte l'unité } 4 \\
\text { à Mitoc-Malu Galben } \\
\text { AlTecte l'unitć } 12 \\
\text { à Molodova V }\end{array}$ & & \\
\hline " ćpisode intcrstadiaire" & $\begin{array}{c}\text { " interstade de Cerman } " \\
\text { = (?) } \\
\text { interstade MG4 }\end{array}$ & $\begin{array}{l}\text { enregistré à } \\
\text { Mitoc-Malu Galben }\end{array}$ & $\begin{array}{l}\text { enregistré ả } \\
\text { Nitra-Ccrman }\end{array}$ & $\vdots$ \\
\hline " ćpisode intcrstadiaire" & $\begin{array}{c}\text { interstade MCi6 } \\
\quad= \\
\text { interstade de l'avlov }\end{array}$ & $\begin{array}{c}\text { enregisré à } \\
\text { Molodova V } \\
\text { Mitoc-Malu (ialben }\end{array}$ & $\begin{array}{l}\text { enregistré ả } \\
\text { WillendorI } \\
\text { l'avlov }\end{array}$ & 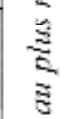 \\
\hline $\begin{array}{l}\text { Refroidissement } \\
\text { drastique }\end{array}$ & limite pléniglaciaire moyen & $\begin{array}{l}\text { iglaciaire supéricur } \\
\text { affecte l'unité } 7 \\
\text { ả Mitoc-Malu (ialhen } \\
\text { Allecte l'unité } 10 \\
\text { à Molodova V }\end{array}$ & & \multirow[t]{2}{*}{ 气 } \\
\hline " épisode interstadiaire" & interslade MCi9 & $\begin{array}{c}\text { Enregistré à } \\
\text { Mitoc-Malu (ialhen }\end{array}$ & & \\
\hline
\end{tabular}

Tableau 3 - Quelques épisodes climatiques enregistrés dans les gisements ouest et est carpatiques par des "phénomènes marqueurs ".

Les épisodes de refroidissement sont signalés sur fond gris clair, les épisodes de réchauffement sur fond gris foncé (d'après Haesaerts et al. 2004)

Table 3 - Some climatic episodes registered by horizon markers in sites west and east of the Carpathians. Cooling episodes are indicated a light gray background; warming episodes by a dark gray background (after Haesaerts et al. 2004).

concernent le Castelperronien et l'Aurignacien, prennent fin lors de la première phase gravettienne à climat de type interstadiaire. La biozone qui a permis d'identifier cette phase climatique caractérise un environnement qui, pour le début du Paléolithique supérieur, représente un cas unique dans l'état actuel de nos connaissances. Ceci conduit à retenir l'hypothèse selon laquelle l'horizon biostratigraphique correspondant (la biozone 6) est un horizon marqueur de phénomènes climatiques à large portée géographique et qu'il doit permettre d'établir des liens chronologiques entre gisements de l'ensemble de l'Europe non méditerranéenne.

A la période d'instabilité contemporaine du début du Paléolithique supérieur, succède une période au climat, semble-t-il, moins contrasté au cours de laquelle le froid et surtout la sécheresse s'accentue plus ou moins régulièrement. La phase dite de transition fait le lien entre les deux. Elle correspond à la biozone 7 et marque une limite entre deux entités temporelles au cours desquelles l'amplitude des variations climatiques paraît d'intensité bien différente (fore auparavant, faible ensuite). Peut-on paralléliser cette limite avec celle qui sépare le Pléniglaciaire moyen du Pléniglaciaire supérieur en Europe du nord et en Europe centrale (tabl. 2) (Mangerud et Berglund 1978 ; Haesaerts et al. op. cit.) ? Cette question est envisagée plus loin.

\section{LES EVENEMENTS-MARQUEURS EN EUROPE CENTRALE}

Dans la vallée du Danube, à l'ouest, comme à l'est des Carpates (fig. 1), ont été menées des recherches chronostratigraphiques intéressant notamment les ensembles gravettiens. Les auteurs insistent sur le fait qu'ils ont pu établir une séquence stratigraphique globale pour le bassin moyen du Danube et le domaine est-carpatique (Haesaerts et al. 2004).

Ces corrélations ont pu être établies sur la base de " marqueurs stratigraphiques " repérables dans plusieurs sites d'une même région ainsi que dans des régions différentes. Dans le domaine est-carpatique, selon ces auteurs, deux évènements se sont exprimés de façon particulière. Le premier correspondrait au refroidissement drastique qui " conclut le Pléniglaciaire moyen ". Il est représenté par " un épais gley de toundra " notamment dans les gisements de Mitoc-Malu Galben en Roumanie et de Molodova $V$ en Ukraine. A Mitoc, ce gley affecte l'unité 7 qui contient les premiers ateliers gravettiens ; à Molodova $V$, il affecte l'unité 10 qui contient les niveaux gravettiens 10 et 9 (Haesaerts et al. op. cit., p. 40). Le second marqueur stratigraphique est aussi un épais gley de toundra qui se développe dans les formations loessiques du 


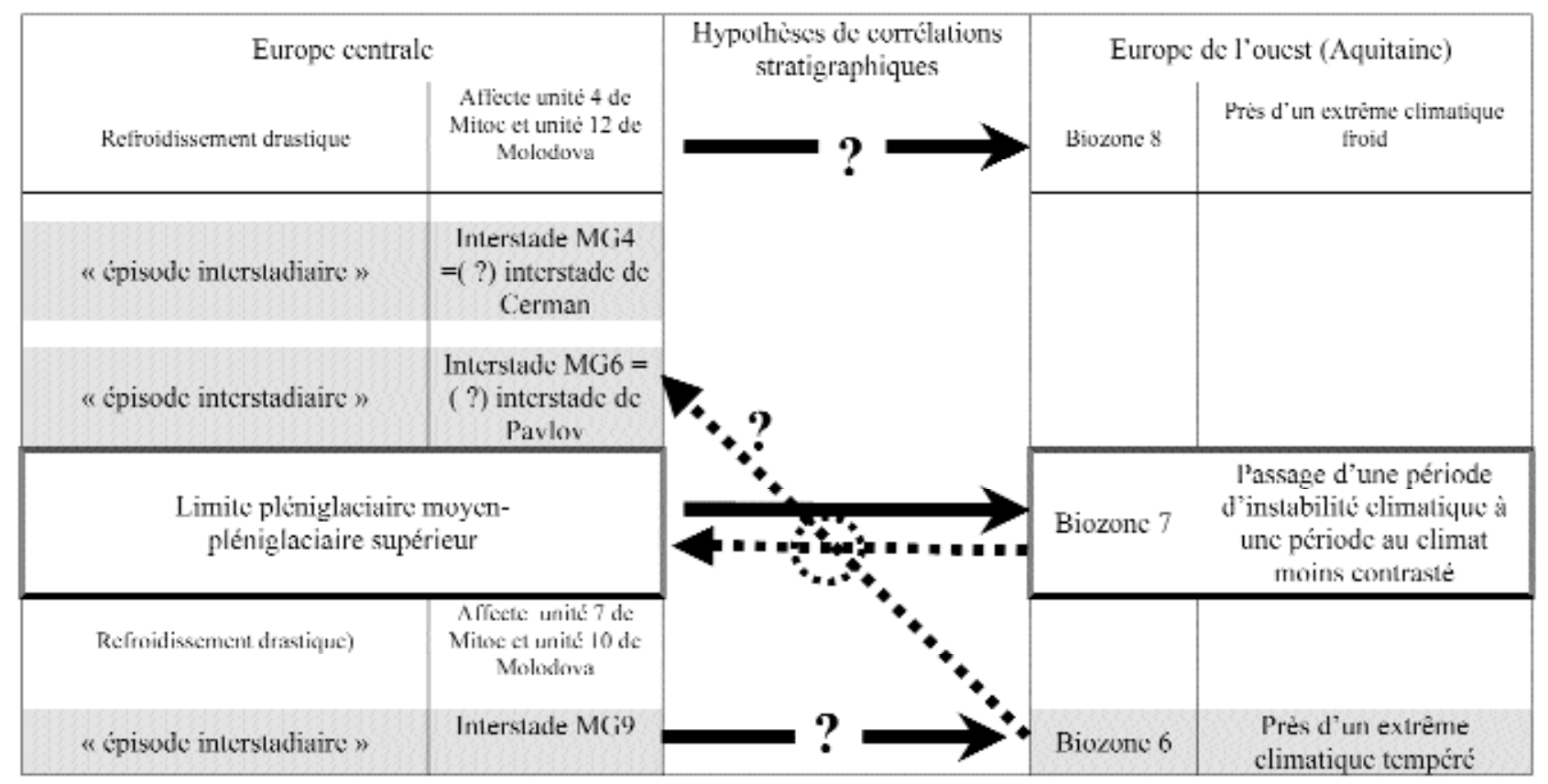

Tableau 4 - Hypothèses de corrélations stratigraphiques entre gisements avec gravettien d'Europe centrale et d'Europe de l'ouest.

Flèches pointillées : priorité à la corrélation entre la période de formation de la biozone 6 et un épisode interstadiaire. Flèches pleines : priorité à la corrélation entre la limite pléniglaciaire moyen-pléniglaciaire supérieur et la période de formation de la biozone 7.

Table 4 - Hypothesized stratigraphic correlations among central and western European Gravettian sites. Dashed arrows: priority given to the correlation between biozone 6 and an interstadial episode. Solid arrows: priority given to the correlation between biozone 7 and the middle and late pleniglacial.

Pléniglaciaire supérieur. A Mitoc-Malu Galben, il affecte l'unité 4 et à Molodova $V$ l'unité 12 ; toutes les deux livrent du Gravettien à pointes à cran (tabl. 3).

De part et d'autre des Carpates, l'événement qui sert de lien entre les gisements, est un "épisode interstadiaire " qui serait enregistré " à la base de la couverture loessique du pléniglaciaire supérieur à Molodova $V$ et à Mitoc " ainsi que dans le bassin moyen du Danube à Willendorf en Autriche et à Pavlov en Moravie ("Interstade de Pavlov ") (Haesaerts et al. op. cit. p. 43). II s'agit de l'Interstade MG 6» (lisible à la base de l'unité 6 de Mitoc-Malu Galben et de l'unité 11 de Molodova V, avec gravettien dit " moyen ") qui correspondrait donc au même événement que l'Interstade de Pavlov". Le Pavlovien, " faciès régional spécifique du gravettien européen ", serait encore représenté à Pavlov ainsi qu'à Willendorf II lors de ce " bref épisode interstadiaire " (Otte et Noiret 2004 p. 13).

Bien que n'ayant pas été relevés nommément par $P$. Haesaerts et al. (2004) pour l'établissement de liaisons entre l'est et l'ouest des Carpates, d'autres " épisodes interstadiaires " ont été repérés de part et d'autre de ce massif montagneux. Citons, à l'est, l'Episode MG9" de Mitoc-Malu Galben qui semble être intervenu juste avant les premières manifestations gravettiennes et l'Episode MG8', apparemment moins marqué. A l'ouest, l'Episode positif de Cerman" (de Nitra Cerman en Slovaquie) pourrait correspondre à l'Interstadiaire MG4» repéré du côté est avec Gravettien à pointes à cran (Haesaerts et al. 2004, fig. 7) (tabl. 3).

\section{QUELS LIENS CHRONOSTRATIGRAPHIQUES ENTRE LES SÉQUENCES GRAVETTIENNES DE L'OUEST ET L'EST DE L'EUROPE ?}

Lors des tentatives de corrélations, nous avons utilisé les éléments suivants :

Pour l'ouest de l'Europe :

1) la biozone 6 en tant que marqueur biostratigraphique d'un environnement de type interstadiaire ;

2) la biozone 7 comme marqueur "séparateur " de deux grands ensembles stratigraphiques correspondant à deux grandes périodes climatiques.

Pour l'Europe centrale :

- les niveaux marqueurs de l'épisode interstadiaire MG6.

A la fois pour l'Europe de l'ouest et l'Europe centrale : 1) la chronologie des événements présentés dans le tableau 3 ; 
2) le fait que la biozone 7 peut correspondre au passage du Pléniglaciaire moyen au Pléniglaciaire supérieur.

En accordant la priorité à l'évènement marqueur de l'ouest de l'Europe (i.e. la biozone 6 qui témoigne d'une ambiance interstadiaire «sub-tempérée»), plusieurs possibilités de corrélations apparaissent. On peut placer le point d'ancrage au niveau de MG4, de MG6 ou de MG9, voire même de MG8 (non indiqué sur le tabl. 4). La logique voudrait que soit utilisé en priorité, pour l'Europe centrale, le marqueur stratigraphique qui a permis de faire le lien entre les séquences ouest et est carpatiques, à savoir " l'épisode interstadiaire ... enregistré à la base de la couverture loessique du Pléniglaciaire supérieur " (interstade MG6) (Haesaerts et al. op. cit. p. 43) et qu'il soit corrélé avec la biozone 6. Mais alors la limite Pléniglaciaire moyenPléniglaciaire supérieur ne correspondrait pas à la biozone 7 , hypothèse que l'on privilégie (tableau 4 et cf. supra).

En revanche, en accordant la priorité à la limite Pléniglaciaire moyen-Pléniglaciaire supérieur et en supposant qu'elle se place au moment où s'est formée la biozone 7 , alors la biozone 6 pourrait correspondre à MG9 (ou MG8 ?). Quant à la biozone 8 , elle pourrait être l'équivalente de l'épisode de refroidissement drastique qui affecte l'unité 4 à Mitoc, et l'unité 12 à Molodova V (tableau 4). Cette hypothèse est supportée par les données car elle ne nécessite pas l'introduction de distorsions dans les successions stratigraphiques. Elle conduit cependant à minimiser l'importance de l'événement climatique responsable de l'originalité des associations fauniques de la biozone 6 dans la mesure où, en Europe centrale, cet événement serait un "épisode interstadiaire " banal qui ne se différencie pas des autres.

Les éléments qui justifient cette hypothèse semblent donc encore assez mal assurés et chaque lien tracé mériterait d'être conforté par d'autres arguments, notamment d'ordre biologique. Ceci n'a pu être fait à ce jour et c'est vers les datations radiométriques que nous nous sommes toumés pour rechercher d'éventuels arguments susceptibles de venir appuyer notre proposition.

\section{LES DATATIONS RADIOMÉTRIQUES}

Si la datation du Gravettien le plus ancien d'Europe centrale (Couche 5 de Willendorf datée de 30500 BP Haesaert et al. 2004, p. 44) n'est retenue que par certains auteurs, il semble y avoir un consensus sur le fait que les datations des ensembles gravettiens se situent pour la plus grande partie entre 28000 et 22000 BP (Jöris et Weninger 2004, fig.1).

Dans l'ouest de l'Europe, les ensembles livrant du Gravettien occupent la même aire temporelle. Ainsi, pour les gisements aquitains cités dans ce travail, les datations s'échelonnent de 29000 BP à 21000 BP (quand on prend en compte une marge d'incertitude de 1) (fig. 3).
Cependant, lorsque l'on tente de situer, dans cet intervalle, les périodes de formation de chacune des biozones reconnues en Aquitaine, on est surpris par le fort chevauchement des datations pour des biozones qui, rappelonsle, sont stratigraphiquement superposées. Si on élimine de la biozone 6 les strates qui ont donné les sept datations les plus récentes (toutes concernent le gisement de La Ferrassie), le recouvrement est moindre et on voit se dessiner une succession temporelle des périodes de formation ${ }^{5}$. Ces sept datations étant éliminées, à la biozone 6 comespondraient des datations s'échelonnant de 29070 à $25550 \mathrm{BP}$, à la biozone 7 une seule date de 27400 à 25600 et à la biozone 8 une plage allant de 27170 à 22020 BP (toujours en années ${ }^{14} \mathrm{C}$ et en prenant en compte l'intervalle de confiance à $1 \sigma$ ). Sur la figure 3 , en vis-à-vis de celles des trois biozones, ont été représentées les datations des ensembles gravettiens d'autres gisements aquitains : Grotte XVI (une datation pour la couche Abc), LaugerieHaute (une datation pour la couche 36) et Pataud (14 datations pour les couches 2 à 5). Les implications de chronologie relative que l'on peut tirer du simple examen de cette figure ne remettent pas en cause la position stratigraphique proposée ci-dessus pour ces ensembles relativement à celle des biozones 6,7 et 8 (cf. tabl. 2).

Voyons maintenant comment se situent les ensembles d'Europe de l'ouest relativement à ceux d'Europe centrale. Dans la séquence chronologique de l'Europe centrale, les datations proposées pour les événements " marqueurs" cités plus hauts sont les suivantes:

- l'épais gley de toundra observé dans les formations du Pléniglaciaire supérieur (qui affecte les unités 4 de Mitoc et 12 de Molodova) a été " daté autour de 23000 BP " (Haesaerts et al. op. cit. p. 41) ;

- I'Interstade MG4 est daté aux alentours de 23700 BP à Molodova V (Haesaerts et al. op.cit. p. 41) ;

- l'Interstade MG6 (qui correspondrait à l'interstade de Pavlov) est daté vers 25500 BP à Molodova V (Haesaerts et al. op.cit. p. 41) ;

- l'épais gley de toundra qui correspond au refroidissement drastique concluant le Pléniglaciaire moyen (II affecte les unités 7 de Mitoc et 10 de Molodova) a été daté à Molodova entre 26640 et 25760 (Haesaerts et al. op.cit. p. 40) ;

- l'Interstade MG8 est daté de 27500 BP (Haesaerts et al. op.cit. p. 40) ;

- l'Interstade MG9 est daté 28500 BP ; il correspondrait à I'horizon humifère de la sous unité 10-3 de Molodova datée entre 28730 et 27700 BP (Haesaerts et al. op.cit. p. 40).

En conséquence, la limite entre le Pléniglaciaire moyen et le Pléniglaciaire supérieur se placerait vers 25700 BP. La mise en regard des dates ouest et centre-européennes (tabl. 5) accepte la proposition de corrélation dite "logique " : la biozone 6 se serait formée au moment où se manifestait soit l'Interstade MG9 soit l'Interstade MG8, la biozone 7 au moment du passage du Pléniglaciaire moyen au Pléniglaciaire supérieur et la biozone 8 posté-

(5) Les problèmes concernant le site de La Ferrassie ont déjà été largement discutés et nous n'y reviendrans pas ici : rappelons simplement que quelques gisements du Périgord ont fait l'objet de révisions stratigraphiques et de réflexions sur leurs processus de formation ce qui a conduit, notamment pour le site de La Ferrassie, à la mise en évidence de la non homogénéité chronologique des archéostrates (Delpech 2007 ; Delpech et Rigaud 2001 ; Texier 2001). 


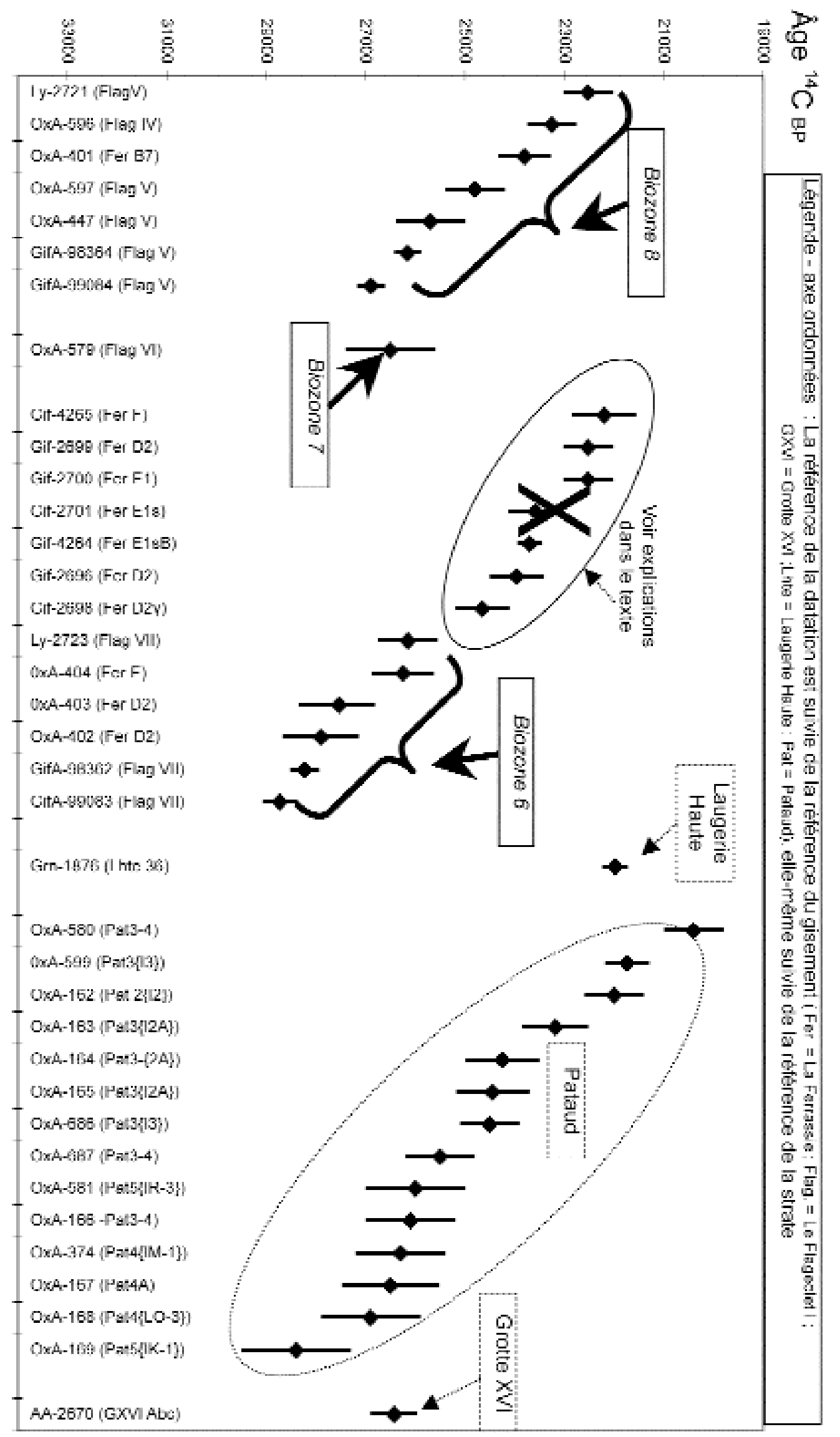




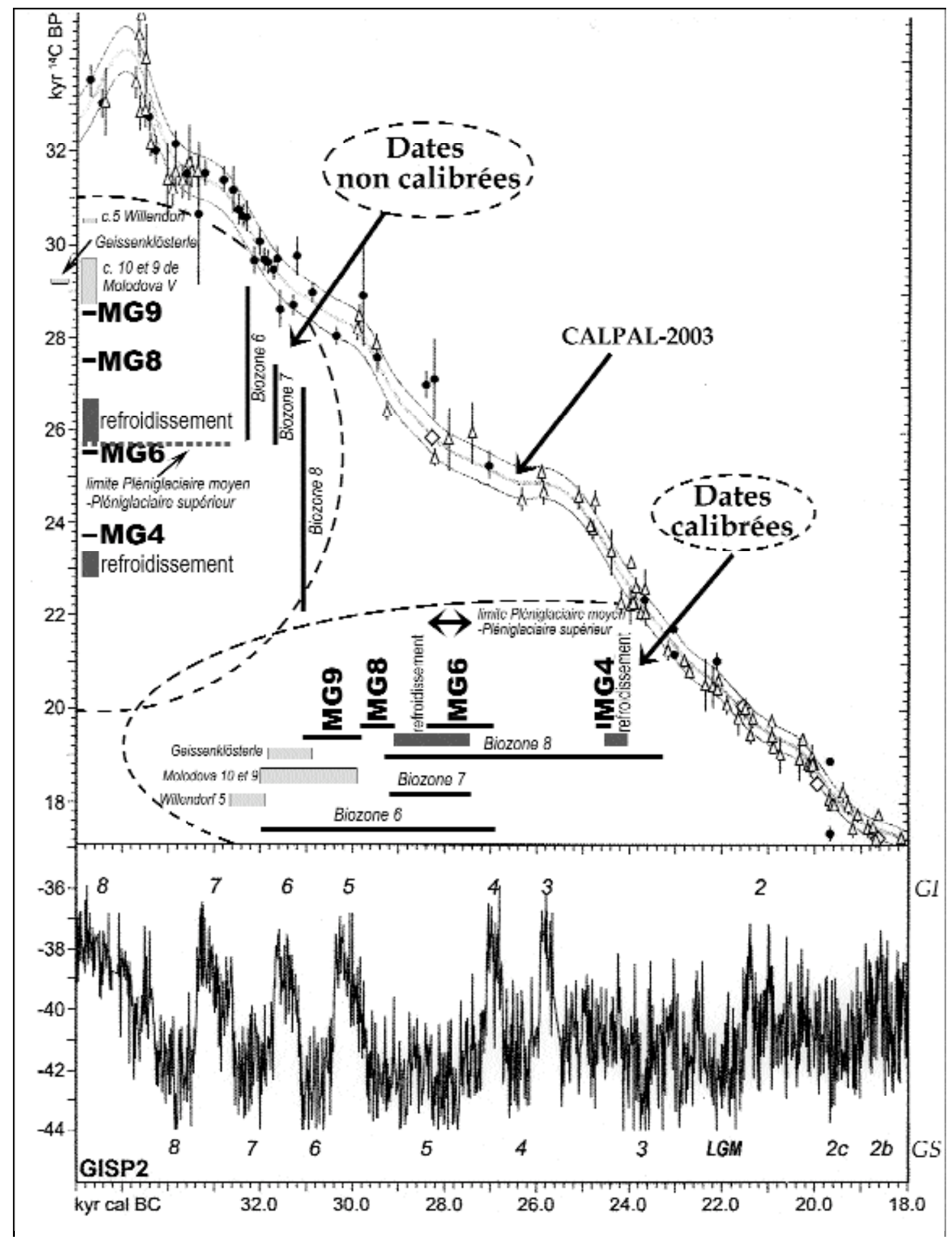

Figure 4 - (d'après Jöris et Weninger, 2004, fig. 3, modifiée) : Âges de quelques événements jalonnant l'époque gravettienne en Kans BP et en Kans cal BC (courbe de calibration CALPAL-2003). Relation avec la courbe 180 de la carotte de glace GISP2 du Groenland. Les interstades GI sont les interstades "Dansgaard-Oeschger"; parmi les stades glaciaires GS, le stade 5 correspondrait à l'événement de Heinrich 3 (cf. Grousset 2001). Voir explications dans le texte.

Figure 4 - Ages of selected events that mark the Gravettian epoch, in thousands of radiocarbon years BP and thousands of calibrated years BC (CALPAL-2003 calibration curve). The relationship with the 180 curve from the Greenland GISP2 ice cap is also shown. The Gl interstadials are the "Dansgaard-Oeschger" interstadials; among the glacial stadials GS, stage 5 corresponds to Heinrich event 3 (cf. Grousset 2001). See text for explanation. 


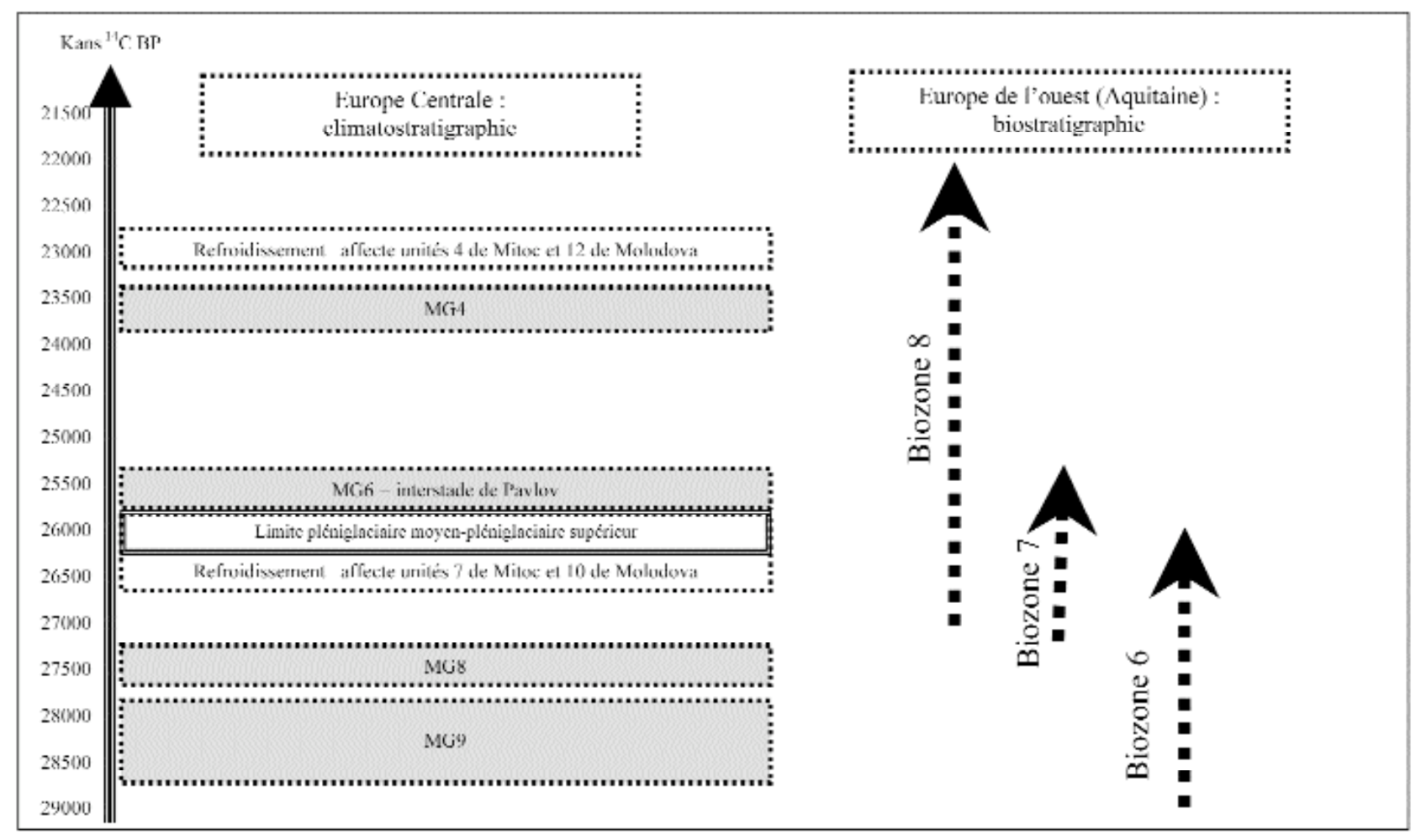

Tableau 5 - Chronostratigraphie de quelques ensembles gravettiens d'Europe centrale et d'Europe de l'ouest. L'épaisseur des aires comme la longueur des flèches reflète non la durée de formation des strates mais des incertitudes sur la position chronologique des couches concernées.

Table 5 - Chronostratigraphy of selected western and central European Gravettian assemblages. The height of the boxes and length of the arrows reflects the degree of uncertainty of the chronological position of the strata involved (and not the duration of those strata).

rieurement, notamment au moment où les unités 4 de Mitoc et 12 de Molodova se mettaient en place.

Un simple examen de la figuration de CALPAL-2003 (Joris et Weninger 2004, fig. 3) montre qu'il est théoriquement possible de proposer des corrélations avec les événements climatiques mis en évidence notamment dans les carottes glaciaires. On sait, cependant, que les dates calibrées ne sont pas des fonctions arithmétiques des dates ${ }^{14} \mathrm{C}$. On constate en effet qu'au-delà de 24000 BP, la courbe de calibration (avec ses courbes enveloppes) fluctue assez fortement (fig. 4). Ainsi, à côté d'un " plateau " qui se développe au niveau de $25000 \mathrm{BP}$ (qui pourrait correspondre à environ 1500 années "réelles"), la courbe montre de fortes pentes à plusieurs niveaux : autour de 30000 BP, 27000 BP, 23000 BP. Ceci signifie qu'à des âges ${ }^{14} \mathrm{C}$ très semblables peuvent correspondre des âges réels très différents. On note également une tendance à l'inversion vers $33000 \mathrm{BP}$, c'est-à-dire qu'une date ${ }^{14} \mathrm{C}$ BP peut correspondre à deux âges réels très différents ou, pour dire les choses autrement, que l'ordonnancement temporel suggéré par plusieurs dates ${ }^{14} \mathrm{C}$ $\mathrm{BP}$ peut aller en sens inverse de la réalité. Par exemple, il est possible qu'un ensemble daté de $33000 \mathrm{BP}$ soit en fait plus jeune qu'un ensemble daté de 32500 BP. En outre, en deçà de 24000 BP, il existe plusieurs courbes de calibration et, à l'heure actuelle, il n'y a pas de consensus pour choisir l'une plutôt que l'autre (Bard et al. 2004). Ainsi, selon la courbe choisie, l'âge d'un rhinocéros de la grotte Chauvet, daté de $31000 \mathrm{BP}$, peut correspondre à 33000,35500 ou 38000 années cal BP.

A ce jour, l'utilisation des datations (calibrées ou non) ne peut pas, à elle seule, servir de base à l'établissement de corrélations. Mais, avons-nous des moyens de contrôle voire de validation? Pouvons-nous vérifier, par exemple, que les datations permettent de situer les effets différents d'un même évènement dans la même tranche de temps ? Actuellement, on sait que, d'une part, les biozones correspondent à des climatozones et que, d'autre part, des liens ont été tracés entre la courbe de calibration CALPAL-2003 et des courbes climatiques, notamment celle dérivée de GISP2. On peut par exemple chercher à vérifier si, lors de la période de formation de la biozone 6 , les glaces ont bien enregistré un climat de type interstadiaire. Cet essai montre, en fait, que cette biozone s'est formée à l'intérieur d'une longue période qui a vu se manifester non seulement les interstades GI 6 et GI 5 mais aussi les stades GS 6 et GS 5 de GIPS 2 (fig. 4). 
Cet exemple, parmi d'autres, montre la difficulté, voire l'impossibilité, d'utiliser les datations (calibrées ou non) pour l'établissement de fines corrélations temporelles. On ne peut, encore aujourd'hui, se fonder sur les seules dates numériques pour établir une chronologie relative des événements propres à l'environnement et à l'Homme du début du Paléolithique supérieur. Loin de nous cependant l'idée d'une inutilité des datations radiométriques car, dans le domaine chronologique qu'elles couvrent, elles s'avèrent indispensables à tous les niveaux des travaux de recherche. Elles sont ici utilisées pour contrôler une hypothèse et nous aident, en outre, à définir la maille chronologique à laquelle il convient de travailler ou en deçà de laquelle il ne faut pas descendre (Delpech et Rigaud 2001 ; Delpech 2002).

\section{CONCLUSIONS}

La période gravettienne a duré plusieurs milliers d'années et l'aire géographique dans laquelle s'est développé le phénomène gravettien s'étend au delà de l'Europe. Dans ce travail, nous avons voulu apporter notre contribution à l'ordonnancement d'assemblages en proposant un classement chronologique de quelques ensembles stratigraphiques livrant des séries rapportées à ce technocomplexe. Cette démarche a nécessité l'emploi de méthodes relevant de la stratigraphie. Celles-ci ont été appliquées à l'échelle régionale d'abord, à une échelle géographique plus large ensuite. Les datations radiométriques ont été utilisées dans un second temps pour contrôler les hypothèses établies.

Nous avons ainsi montré qu'en Aquitaine, le Gravettien couvre apparemment une plage temporelle relativement large comportant des épisodes climatiques contrastés. Au moins trois périodes ayant entraîné la mise en place d'environnements biologiques particuliers se sont succédées. Dans la chronologie numérique, elles se situent entre 29000 et 22000 BP. II serait d'ailleurs plus juste de dire que ces périodes gravettiennes se rangent dans une plage chronologique s'étalant de part et d'autre de 25000 BP sur au moins trois milliers d'années ${ }^{14} \mathrm{C}$. Le phénomène gravettien a atteint l'Aquitaine septentrionale au moment où se mettaient en place des conditions climatiques relativement clémentes, de type interstadiaire, ce qui a favorisé le développement des essences de forêt tempérée et des ongulés inféodés à ce milieu. Le phénomène culturel gravettien s'est poursuivi en Aquitaine, sous un climat plus frais, puis sous des conditions de steppes froides et sèches. II faut rappeler cependant que la séquence biostratigraphique de référence, ne repose encore que sur peu de gisements. Aussi il n'est pas impossible que la prise en compte de nouvelles données conduise à complexifier la biozonation présentée ici et, par voie de conséquence, la zonation climatique des strates gravettiennes. Cependant, dès à présent, un certain nombre d'entités stratigraphiques peuvent être considérées en fonction de leur ancienneté relative, c'est-à-dire qu'il est possible de raisonner, d'interpréter les diverses manifestations de variabilité en prenant en compte le facteur temps.
Les essais de corrélations effectués entre gisements d'Europe de l'ouest et d'Europe centrale, s'ils n'ont pas permis de grandes avancées, n'ont cependant pas été tout à fait vains. Ainsi, la mise en relation de la biozone 6 d'Aquitaine et l'épisode interstadiaire MG9 d'Europe centrale semble raisonnable et en accord avec les datations numériques existantes.

Enfin, ce travail montre qu'il n'est pas pertinent de tenter d'établir des corrélations fines sur la base des seules datations radiocarbones, qu'il s'agisse de dates calibrées ou non. Les plages de datation considérées (qui, d'ailleurs, contiennent ou non la " date véritable ") sont le plus souvent larges pour les périodes qui nous préoccupent ici. Cette vérité mérite d'être rappelée car on est souvent surpris de l'usage qui est fait de ces données. Par exemple, Jöris et Weninger (2004), en s'appuyant uniquement sur des datations, émettent l'hypothèse selon laquelle, entre $27800 \mathrm{BP}$ et $17000 \mathrm{BP}$, les hommes ont évité la Moravie pendant les interstadiaires. Or, une telle hypothèse implique un degré de résolution temporelle très élevé si l'on tient compte du nombre d'interstades enregistrés par exemple dans GISP2 (Grousset 2001). En revanche, la date apparemment " trop ancienne " obtenue pour le Gravettien stratigraphiquement le plus ancien d'Europe centrale (Haesaerts et al. 2004 p. 35 et 44) (couche 5 de Willendorfdatée de 30500 BP) ne nous semble pas vraiment gênante. En effet, comme on vient de le voir, les incertitudes de la méthode impliquent que cette date appartient en fait à la même tranche de temps que le Gravettien des couches 10 et 9 de Molodova V (daté entre 29650 et 28730 BP) ou que le plus ancien Gravettien de la Geissenklösterle dans le Jura souabe (29200 BP d'après Haesaerts op. cit. p. 44) (fig. 4). Comme nous l'avions conclu lors de la présentation orale faite lors du colloque de juillet 2004 , l'établissement de corrélations à grande distance nécessite

1) d'appliquer les principes de la stratigraphie,

2) de contrôler l'homogénéité chronologique des échantillons pris en compte,

3) de croiser les méthodes et

4) de les utiliser en deçà de leur potentiel (apparent ?) de précision.

\section{Remerciements}

Nous remercions le Professeur Donald Grayson pour ses traductions et ses remarques judicieuses qui ont permis d'améliorer sensiblement le manuscrit.

\section{BIBLIOGRAPHIE}

ALAUX J.-Fr. 1969 - Note préliminaire sur l'abri périgordien des Battuts (Tarn). Bulletin de la Société préhistorique française, 66 , p. 10-15

BARD E., MENOT-COMBES G. et DELAYGUE G. 2004 Des dates fiables pour les 50000 demières années. Pour la Science, 42, p. 54-59. 
BORDES Fr. 1958 - Nouvelles fouilles à Laugerie-HauteEst. Premiers résultats. L'Anthropologie, t. 62, p. 205-244.

BORDES Fr. 1968 - La question périgordienne. La Préhistoire, problèmes et tendances. Edition du CNRS, p. 59-70.

BORDES Fr. et SONNEVILLE-BORDES D. de 1966 Protomagdalénien ou périgordien VII ? L'Anthropologie, t. 70, p. 113 à 122.

BOUCHUD J. 1975 - Etude de la faune de l'abri Pataud. In H.L. Movius, éd. :"Excavation of the abri Pataud". American School of prehistoric research bulletin, 30, p. 69-153.

DELPECH Fr. 1983 - Les faunes du Paléolithique supérieur dans le Sud-Ouest de la France. Cahiers du Quaternaire, 6, Editions du CNRS : 453 p., 72 fig., 147 tabl., 6 pl.

DELPECH Fr. 1986 - Les Rennes du grand abri de Laugerie-Haute en Dordogne (fouilles F. Bordes). Arqueologia, Porto, 13, p. 66-71.

DELPECH Fr. 1999 - Biomasse d'Ongulés au paléolithique et inférences démographiques. Paléo, 11, p.19-42, 10 fig., 4 tabl.

DELPECH Fr. 2002 - Apports des datations en Archéologie préhistorique. In Compte-Rendu de la Table ronde : "Archéologie, patrimoine culturel et datation par le carbone 14 par spectrométrie de masse par accélérateur ", 22 mars 2002, Centre de Recherche et de Restauration des Musées de France. Ministère de la Culture et de la Communication, CNRS, p. 16-18.

DELPECH Fr. 2003 - L'environnement animal des européens au Paléolithique supérieur. In $\mathrm{R}$. Desbrosse et $\mathrm{A}$. Thévenin (eds) : "Préhistoire de l'Europe. Des origines à l'Age du Bronze". Actes des Congrès nationaux des Sociétés historiques et scientifiques. 125 ème, Lille 2000, Editions du C.T.H.S., Paris, p. 271-289.

DELPECH Fr. 2007 - Le grand abri de La Ferrassie, source de réflexion sur la biostratigraphie d'un court moment du Pléistocène. In Arts et culture de la Préhistoire. Hommage à Henri Delporte. R. Desbrosse et A. Thévenin éd. p. 303304, 4 fig., 2 tabl.

DELPECH Fr. 2008 - Recherches sur la mobilité humaine au Pléistocène. In J. Gomez de Soto éd. " La notion de mobilité dans les Sociétés préhistoriques. Diffusion des idées, déplacements de population ". Publication électronique CTHS.

DELPECH Fr., GRAYSON D. K et RIGAUD J.-Ph. 2000 Biostratigraphie et paléoenvironnements du début du Würm récent d'après les grands mammifères de l'abri du Flageolet I (Dordogne, France). Paléo, 12, p. 97-126, 18 fig., 32 tabl.

DELPECH Fr. et RIGAUD J.-Ph. 2001- Nouveaux apports des datations en Archéologie préhistorique. In Barrandon
J.-N., Guibert P., Michel V. (Eds) Datation. XXle Rencontres internationales d'Archéologie et d'histoire d'Antibes. Editions APDCA, p. 315-331, 7 fig.

DELPORTE H. 1984 - L'Aurignacien de La Ferrassie. In H. Delporte ed. : "Le grand abri de La Ferrassie, fouilles 19681973". Etudes Quatemaires, 7, p. 145-234

GRAYSON D. K. et DELPECH Fr. 2003 - Ungulates and the Middle-to-Upper Paleolithic transition at Grotte XVI (Dordogne, France). Journal of Archaeological Science, 30 , p. $1633-1648$

GROUSSET F. 2001 - Les changements abrupts du climat depuis 60000 ans. Quatemaire, 12, (4), p. 203-211.

HAESAERTS P., BORZIAK I., CHIRICA V., DAMBLON F., KOULAKOVSKA L. et VAN DER PLICHT J. 2003 - The East Carpathian loess record: a reference for the Middle ans Late Pleniglacial stratigraphy in Central Europe. Quatemaire, 14 (3), p. 163-188

HAESAERTS P., BORZIAK I., CHIRICA V., DAMBLON F. et KOULAKOVSKA L. 2004 - Cadre stratigraphique et c h ronologique du gravettien en Europe Centrale. In Jirí A. Svoboda and Lenka Sedlárková éds : The Gravettian along the Danube. The Dolni Vestonice Studies, 11, p. 33-56

HEDBERG H. 1979 - Guide stratigraphique intemational. Classification, terminologie et règles de procédure. Doin édit., Paris, 233 p.

JÖRIS O. et WENINGER B. 2004 - Coping with the cold : on the climatic context of the Moravian Mid Upper Palaeolithic. In Jirí A. Svoboda and Lenka Sedlárková éds : The Gravettian along the Danube. The Dolni Vestonice Studies, 11 , p. $57-70$.

LAVILLE H., TURON J.-L., RAYNAL J.-P., DELPECH F., PAQUEREAU M.-M., PRAT F. et DEBENATH A. 1983 Histoire paléoclimatique de l'Aquitaine et du Golfe de Gascogne depuis le dernier interglaciaire. Actes Colloque AGSO, Bordeaux, 1983 : "Paléoclimats" et CNSR, Cahiers du Quatem aire, n spécial, p. 219-241.

MANGERUD J. et BERGLUND B.E. 1978 - The subdivision of the Quatemary of Norden : a discussion. Boreas, Oslo, 7 (3), p. 179-181.

OTTE M. et NOIRET P. 2004 - Evolution de gravettien au Moyen Danube ? In Ji_í A. Svoboda and Lenka Sedlárková éds : The Gravettian along the Danube. The Dolni Vestonice Studies, 11, p. 8-32.

PEYRONY D. et PEYRONY E. 1938 - Laugerie-Haute près des Eyzies (Dordogne). A rchives de l'Institut de Paléontologie Humaine, mémoire 19, 84 p..

RAMADE Fr. 1984 - Eléments d'écologie fondamentale, McGraw-Hill, Paris, 397 p. 
RIGAUD J.-Ph. 2008 - Les industries lithiques du gravettien du nord de l'Aquitaine dans leur cadre chronologique. Paléo, sous presse.

RIGAUD J.-Ph., LUCAS G. et SIMEK J. 2002 - Cénac et Saint-Julien, Grotte XVI. Bilan scientifique de la région Aquitaine 2001, Direction Régionale des Affaires Culturelles d'Aquitaine, Service régional de l'Archéologie, p. 30-32.
SPIESS A. E. 1979 - Reindeer and caribou hunters. An archaeological study. Academic Press, New York, San Francisco, London, 312 p.

TEXIER J.-P. 2001 - Sédimentogénèse des sites préhistoriques et représentativité des datations numériques. In Barrandon J.-N., Guibert P., Michel V. (Eds) Datation. XXle Rencontres internationales d'Archéologie et d'histoire d'Antibes. Editions APDCA, p. 159-175. 
